\title{
Avaliação gravimétrica dos resíduos de serviços de saúde no município de Rondonópolis (MT) Brasil
}

\section{Evaluation of gravimetric health services waste in Rondonópolis (MT) Brazil}

\author{
Michele Salles da Silva ${ }^{1}$, Patrício Marques de Souza ${ }^{2}$, Ricardo Alves de Olinda ${ }^{3}$, Débora Aparecida Silva Santos ${ }^{4}$, Rosidelma \\ Xavier de Oliveira ${ }^{5}$
}

\begin{abstract}
Resumo: O resíduo de serviço de saúde (RSS) é resíduo proveniente de atividades exercidas em estabelecimentos diferenciados (seja biológico, químico, perfurocortantes e comum produzidos dentro desses locais), que vem aumentando diariamente e que exigem, na maioria das vezes, cuidados especiais desde sua geração a sua disposição final. Este estudo objetivou analisar o gerenciamento dos RSS a partir da avaliação gravimétrica de resíduos de uma instituição hospitalar de Rondonópolis (MT) Brasil. Como metodologia foi realizada uma pesquisa exploratória, descritiva, quantitativa, observacional e longitudinal. Baseada nos Planos de Gerenciamento de RSS entre os anos de 2010 e 2014, foi possível fazer uma análise gravimétrica dos RSS por leito ocupado por dia, com os seguintes resultados: $1,57 \mathrm{Kg}$ em $2010 ; 1,70 \mathrm{Kg}$ em $2011 ; 1,57 \mathrm{Kg}$ em 2012; 1,86Kg em 2013 e 1,98Kg. (leito.ocupado.dia)-1 em 2014; com uma taxa média de 1,73Kg .(leito.ocupado.dia)-1 nos cinco anos analisados. Foram sugeridas propostas de melhoria para o gerenciamento de RSS, uma mudança de atitude com maior comprometimento dos profissionais envolvidos a fim de melhorar o atendimento à saúde da população, bem como a qualidade ambiental, através da redução de geração e segregação adequada dos resíduos.
\end{abstract}

Palavras-chaves: Gerenciamento de resíduos, Taxa gravimétrica de resíduos, instituição hospitalar.

\begin{abstract}
The health service residue (RSS) is residue from activities performed in different venues, which is increasing daily (Whether biological, chemical, sharps and common produced in these locations ), and requiring, in most cases, special care from its generation to its final disposal. This study aimed to analyze the management of RSS from the gravimetric evaluation of waste from a hospital Rondonópolis (MT) Brazil. Methodology as exploratory, descriptive, quantitative, observational and longitudinal research was conducted. Based on the Plans RSS Management between the years 2010 and 2014, it was possible to gravimetric analysis by RSS occupied bed per day, with the following results: $1,57 \mathrm{Kg}$ in $2010 ; 1,70 \mathrm{Kg}$ in $2011 ; 1,57 \mathrm{Kg}$ in $2012 ; 1,86 \mathrm{Kg}$ in 2013 and $1,98 \mathrm{Kg}$ (occupied.bed.day) ${ }^{-1}$ in 2014 ; with an average rate of $1,73 \mathrm{Kg}$. (occupied.bed.day) ${ }^{-1}$ in the five years analyzed. Improvement proposals were suggested for the RSS management, a change of attitude with greater commitment of the professionals involved in order to improve the health care of the population as well as environmental quality, by generating reduction and proper waste segregation.
\end{abstract}

Key words: Waste management, gravimetric rate, hospital institution.

\footnotetext{
* Autor para correspondência.

Recebido para publicação em 07/11/2015; aprovado em 25/12/2015

${ }^{1}$ Enfermeira. Prof a Assistente II do Curso de Enfermagem da Universidade Federal de Mato Grosso - UFMT, doutoranda em Recursos Naturais de

Universidade Federal de Campina Grande - UFCG, e-mail: michelesalles@ufmt.br

${ }^{2}$ Veterinário. Doutor em Clínica Veterinária pela Universidade de São Paulo - USP. Professor e orientador do Programa de Pós-graduação em recursos

Naturais - PPGRN, e-mail: patriciomsouza@ig.com.br

${ }^{3}$ Estatístico. Doutor em Estatística Experimentação Agronômica pela Universidade de São Paulo - USP. Professor da Universidade Estadual da Paraíba -

UEPB, e-mail: ricardo.estat@yahoo.com.br.

${ }^{4}$ Enfermeira. Prof ${ }^{a}$ Adjunto I do Curso de Enfermagem da Universidade Federal de Mato Grosso - UFMT, doutoranda em Recursos Naturais de Universidade Federal de Campina Grande - UFCG, e-mail: deboraasantos@ hotmail.com

${ }^{5}$ Bióloga. Responsável pelo Programa de Gerenciamento de Resíduos Sólidos do Hospital Santa Casa de Misericórdia de Rondonópolis-MT, e-

mail:higienizacao@santacasarondonopolis.com.br
} 


\section{INTRODUÇÃO}

O crescimento das cidades vem ocorrendo atualmente devido ao aumento da busca por oportunidades de emprego e moradias nas grandes cidades (denominado êxodo rural) mesmo enfrentando condições que não atendam as expectativas esperadas. Como consequência desse aumento, muitas vezes desordenado, eleva-se a também a produção de resíduos sólidos, podendo assim gerar inúmeros incidentes/acidentes e problemas de saúde e para o meio ambiente (FONSECA et al., 2013).

Segundo o Panorama dos Resíduos Sólidos no Brasil os resíduos sólidos podem ser divididos em resíduos sólidos urbanos (domiciliares, resíduos de limpeza urbana, comerciais, industriais, eletrônicos e perigosos) resíduos de construção/demolição e os resíduos de serviços de saúde (ABRELPE, 2014).

A quantidade e a qualidade desses resíduos podem variar devido vários fatores, tais como sociais, econômicos, culturais, geográficos, climáticos, ou seja, dependem do poder aquisitivo, dos hábitos e do nível educacional da população de uma mesma região (MOURA et al., 2012).

O resíduo de serviço de saúde (RSS) é resíduo derivado de atividades exercidas por estabelecimento gerador que necessitam de cuidados diferenciados no manejo, exigindo ou não o tratamento prévio antes de sua disposição final (FEAM, 2008).

Além disso, podem ser gerados em todos os serviços relacionados com o atendimento à saúde humana ou animal, inclusive os serviços de assistência domiciliar e de trabalhos de campo; laboratórios analíticos de produtos para saúde; necrotérios, funerárias e serviços em que se realizem atividades de embalsamento (tanatopraxia e somatoconservação); serviços de medicina legal; drogarias e farmácias, inclusive as de manipulação; estabelecimentos de ensino e pesquisa na área de saúde; centros de controle de zoonoses; distribuidores de produtos farmacêuticos; importadores, distribuidores e produtores de materiais e controles para diagnóstico in vitro; unidades móveis de atendimento à saúde; serviços de acupuntura; serviços de tatuagem, dentre outros similares (ANVISA, 2004; CONAMA, 2005).

Devido à grande heterogeneidade desses resíduos vários instrumentos foram criados para ajudar na classificação dos mesmos e a mais utilizada é a baseada nas Resoluções da ANVISA (2004) e do CONAMA (2005), que segregam os resíduos em cinco grupos: Grupo A - infectantes (resíduos com a possível presença de agentes biológicos - vírus, bactérias, fungos), Grupo B - químicos (resíduos que podem apresentar risco à saúde ou ao meio ambiente, dependendo de suas características quanto a inflamabilidade, corrosividade e toxicidade), Grupo C - resíduos radioativo; Grupo D comuns (resíduos com características similares às dos resíduos domiciliares, podendo ser subdivididos em recicláveis e não recicláveis); e, Grupo E - perfurocortantes (objetos e instrumentos contendo cantos, bordas, pontos rígidos e agudos capazes de cortar ou perfurar, utensílios de vidro quebrados). Esta classificação é importante para o correto gerenciamento dos RSS desde sua segregação até destinação final.

O gerenciamento dos RSS é um conjunto de procedimentos de gestão a partir de normativas legais com objetivo de minimizar a produção, bem como gerar um tratamento eficiente, visando a preservação da saúde pública, dos recursos naturais e do meio ambiente. Para que esse gerenciamento seja eficaz é necessário a elaboração de um plano denominado Plano de Gerenciamento de Resíduos de Serviços de Saúde (PGRSS) que deve contemplar várias etapas de manejo, dentre elas a segregação, acondicionamento, identificação, tratamento intermediário, transporte interno, armazenamento temporário, armazenamento externo, coleta e transporte externos, tratamento final e destinação final (COSTA, 2012).

Segundo as Resoluções da Agência Nacional de Vigilância Sanitária (ANVISA) RDC n³06/2004 e do Conselho Nacional de Meio Ambiente (CONAMA) n³58/2005, os RSS são classificados em cinco grupos principais, que necessitam de gerenciamento adequado dentro e fora das instituições de saúde, para tentar reduzir ao máximo sua geração, e, consequente seu descarte na natureza (ANVISA, 2004; CONAMA, 2005).

Esse tipo de resíduo (biológico, químico, perfurocortante e comum produzidos dentro das instituições de saúde), vem crescendo diariamente devido à complexidade dos atendimentos a saúde e utilização de equipamentos/materiais de última geração, além dos cuidados destinados à população, em especial a idosa, que necessita de mais serviços de saúde, logo, maior consumo de materiais e descarte de resíduos.

A preocupação com o gerenciamento adequado dos RSS tem aumentado nos últimos tempos devido o crescente estudo a respeito dos riscos para a população que entra em contato com esse material e o risco que a natureza pode sofrer quando ao impacto ambiental. Várias tecnologias têm sido empregadas no tratamento desses resíduos, porém a forma mais eficaz de redução do mesmo seria a redução da produção desse resíduo, pois quanto menor for a produção, menor será o gasto com o tratamento do mesmo.

Um bom plano de gerenciamento de RSS pode minimizar a produção desse resíduo nas unidades de saúde geradoras, desde que a instituição faça uma ampla divulgação do plano e proporcione a seus funcionários capacitação periódica e educação permanente. Contudo a quantidade gerada de RSS depende de alguns fatores, dentre eles o tipo e tamanho do estabelecimento de saúde; tipo de serviços oferecidos; quantidade de pacientes atendidos; quantidade de leitos da instituição; quantidade de procedimentos médicohospitalares realizados.

Diante do exposto, este estudo teve como objetivo analisar o gerenciamento dos RSS (biológico, químico, perfurocortante e comum) a partir da avaliação gravimétrica de uma instituição hospitalar de Rondonópolis, Mato Grosso, Brasil, dos anos 2010 a 2014. A partir das informações colhidas nos planos de gerenciamento de RSS foi possível fazer uma análise gravimétrica da geração de RSS por leito ocupado por dia, de uma instituição de saúde de Rondonópolis-MT, Brasil, que serviu de parâmetro comparativo com outras instituições de saúde e avaliação do gerenciamento dos RSS da instituição pesquisada.

\section{MATERIAL E MÉTODOS}

Trata-se um estudo exploratório, descritivo, quantitativo, observacional e longitudinal sobre o gerenciamento dos RSS 
de uma instituição hospitalar localizada na cidade de Rondonópolis-MT, sendo esta a $3^{\mathrm{a}}$ maior cidade do estado de Mato Grosso, polo da região Sul de Mato Grosso, que possui uma população aproximada de 202.309 habitantes e com densidade demográfica de 47,0 hab/ $\mathrm{km}^{2}$ (IBGE, 2012). O município é considerado referência para atenção de média e alta complexidade, no setor saúde, seja ambulatorial ou hospitalar, para os 19 (dezenove) municípios que compõem a microrregião Sul Matogrossense (SES/MT, 2015).

O local de estudo foi um hospital referência para maternidade com estrutura híbrida de gestão da saúde, ou seja, baseada no funcionamento simultâneo de atendimento público e privado, que atua complementando as diretrizes do SUS. Consta com 681 profissionais e 205 leitos, divididos entre clínico, cirúrgico, unidades de terapia intensiva (Adulto, Neonatal e Coronária), pediátrico, ginecológico, oncológico e outras especialidades.

Os dados foram coletados no período de janeiro a julho/2015 por meio de entrevistas com os funcionários e avaliação dos Planos de Gerenciamento de Resíduos de Serviços de Saúde da instituição escolhida, dos anos de 2010 a 2014. As informações destacadas a partir da coleta foram a quantidade de RSS produzida ao longo dos anos, de 2010 a 2014, e a variação do número de leitos da instituição no decorrer desse período.

A análise gravimétrica foi realizada baseada no cálculo da taxa de geração de RSS por leito ocupado por dia, na qual foi possível verificar possíveis relações entre as variáveis e organizadas sob forma de gráficos e tabelas. Alguns estudos, como o Manual de Gerenciamento de Resíduos de Serviços de Saúde do Ministério da Saúde, descrevem indicadores ambientais, e dentre eles pode-se citar a Taxa de Resíduos de Serviços de Saúde (BRASIL, 2001), a qual foi trabalhada nessa pesquisa, de forma adaptada, associada a análise gravimétrica de resíduos e apresentada pela seguinte fórmula:

Taxa Gravimétrica de RSS*:

Peso dos RSS Grupos (A+B+D+E) no período $\times 100$

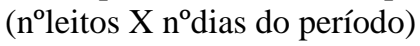

*Adaptado do Manual de Gerenciamento de Resíduos de Serviços de Saúde (BRASIL, 2001)

Nesse sentido, a composição gravimétrica faz parte da análise gravimétrica, que é uma ferramenta essencial podendo propor medidas a serem tomadas com os resíduos desde sua coleta até seu destino final, de uma forma economicamente viável e sempre tendo em vista a sustentabilidade ambiental. Neste estudo a análise gravimétrica será através do cálculo da taxa de RSS/leito/dia, ou seja, a taxa gravimétrica total dos RSS, para servir de base comparativa com outros estudos de instituições de saúde.

\section{RESULTADOS E DISCUSSÃO}

A partir dos PGRSS dos anos de 2010 a 2014 da instituição pesquisada, foi possível descrever a quantidade de RSS (separado por Grupos), e, comparar com a quantidade de leitos que o hospital possui nos respectivos anos, para a realização do cálculo gravimétrico. Esses dados estão demonstrados na Tabela 1.

Tabela 1 - Quantidade do resíduo de serviço de saúde (RSS) durante os anos de 2010 a 2014. Anos

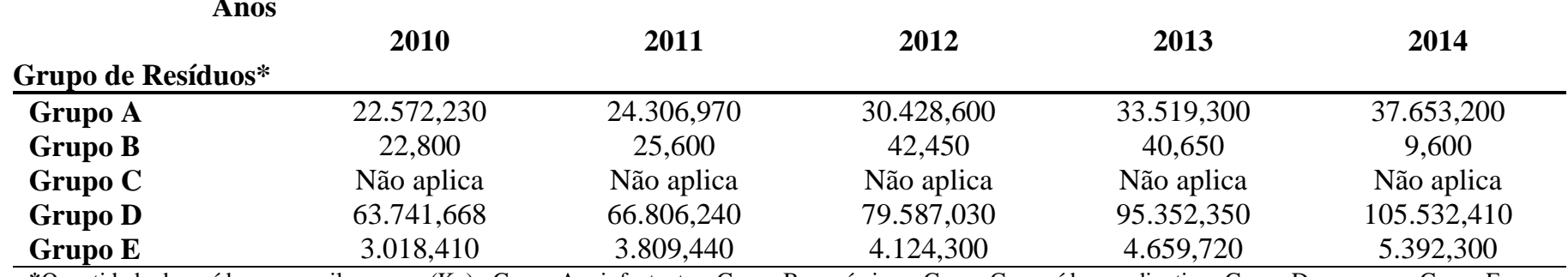

*Quantidade de resíduos em quilogramas (Kg) . Grupo A - infectantes; Grupo B - químicos; Grupo C - resíduos radioativo; Grupo D - comuns; Grupo E perfurocortantes

Realizado pelos autores baseado nos PGRSS dos últimos cinco anos da instituição pesquisada

A partir da Tabela 1 é possível perceber que em todos os anos analisados, os resíduos comuns (Grupo D) foram os que obtiveram maior quantidade em quilogramas $(\mathrm{Kg})$, seguido pelos resíduos do Grupo A, Grupo E e Grupo B, visto não terem sido registrados os dados referentes aos resíduos do Grupo C.

Como a maior quantidade de resíduos encontrada foi a do Grupo D, e estes são classificados como resíduo comum, divididos em recicláveis ou não, acredita-se que para reduzilo seja necessária mais conscientização a respeito de desperdício de alimentos (segundo os não-recicláveis), pois a instituição pesquisada já possui convênio com a cooperativa de reciclagem "Recicla Rondonópolis" que é responsável por destinar todo o papel, plástico e metal do hospital, que sejam classificados como recicláveis.

Associações de reciclagem são iniciativas que desenvolvem ações concretas para reduzir os impactos ambientais, além da geração de renda para a melhoria da qualidade de vida dos associados. O fortalecimento da inclusão social de pessoas, antes desempregadas ou marginalizadas, agora serve de incentivo, facilitando seu trabalho através da segregação dos resíduos de maneira adequada, e, consequentemente uma coleta rentável. Ações participativas da comunidade podem levar a diversas sugestões para a problemática dos resíduos, bem como conscientizar ainda mais as pessoas sobre a importância da reciclagem para a preservação ambiental e redução de doenças, através de medidas educação ambiental (SOUSA JÚNIOR et al., 2008).

A Figura 1 apresenta os valores médios da produção de RSS dos diferentes grupos, em quilogramas, nos cinco anos pesquisados. Os valores percentuais da quantidade dos diferentes grupos de resíduos analisados foram: Grupo $\mathrm{A}=29.696,06 \mathrm{Kg} \quad(25,57 \%) ;$ Grupo $\mathrm{B}=28,22 \mathrm{Kg} \quad(0,02 \%)$, Grupo $\mathrm{D}=82.203,94 \mathrm{Kg}(70,79 \%)$ e Grupo $\mathrm{E}=4.200,83 \mathrm{Kg}$ $(3,62 \%)$. 
Figura 1. Média do resíduo de serviço de saúde (RSS) por Grupos.

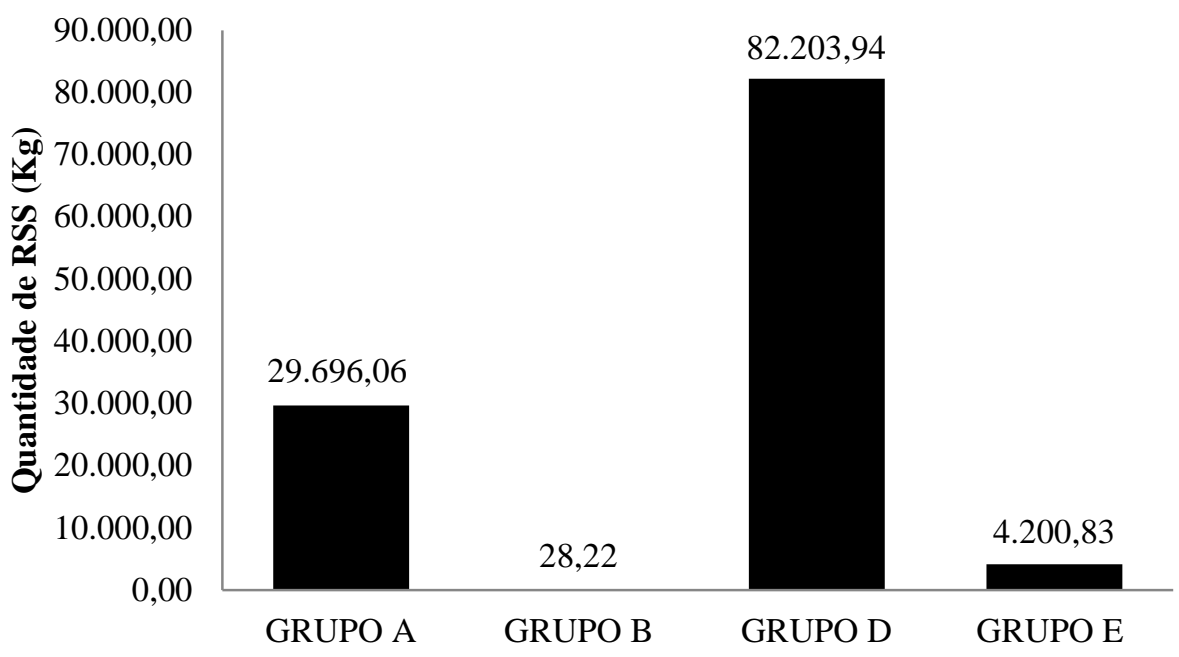

Classificação dos Grupos de RSS

Grupo A - infectantes; Grupo B - químicos; Grupo C - resíduos radioativo; Grupo D - comuns; Grupo E - perfurocortantes

Realizado pelos autores baseado nos PGRSS dos últimos cinco anos da instituição pesquisada

Dentre os principais fatores que interferem na determinação do peso específico aparente dos RSS têm-se: a variedade dos tipos de resíduo (plásticos, papeis, vidros, borracha, panos, materiais orgânicos, fraldas descartáveis usadas, secreções, fluidos em geral); a acomodação dos resíduos no interior dos sacos e o grau de compactação. O descarte de líquidos em lixeiras, embora não seja uma prática correta, é muito comum em algumas unidades de saúde, resultando no aumento da umidade e do peso dos RSS. Um estudo realizado em Vitória-ES descreveu as médias das quantidades de resíduos conforme os grupos: $41 \%$ do Grupo A; $1,5 \%$ do Grupo B; $57 \%$ do Grupo D; e, $0,05 \%$ do Grupo E. Para os pesquisadores a segregação incorreta na fonte de geração é um dos principais fatores responsáveis pelos gastos com os RSS nas instituições hospitalares, e a esta falha mais comum é o acondicionamento de resíduos do Grupo A e D juntos (ADUAN et al., 2014).

Ao comparar esta pesquisa, realizada em RondonópolisMT, com o estudo de Vitória-ES, destaca-se dois grupos de resíduos: Grupo A e Grupo D; contudo o primeiro grupo possui valores superiores em Mato Grosso e o segundo grupo com valores maiores no Espírito Santo.

Acredita-se que a principal diferença esteja na segregação desses resíduos, que poderiam ser reduzidos conforme a aplicação das regulamentações de maneira mais severa nas instituições, que consequentemente, demandariam menor custo financeiro e impacto na natureza. Para Sousa et al. (2015), seguindo a legislação vigente favorece o gerenciamento adequado dos resíduos, utilizando-se de estratégias para o manejo correto desde sua produção até sua produção final, com disposição ambientalmente adequada.

Além da segregação apropriada dos diversos tipos de RSS, a instituição deveria haver mais controle com desperdício de resíduo comum, em especial, os alimentares, visto que o hospital pesquisado oferece refeições para funcionários e pacientes diariamente, logo, quando não consumidos totalmente, geram toneladas de resíduos não reaproveitáveis.

A tão almejada segregação dos resíduos, conforme as normativas nacionais, só será possível quando a instituição tiver implantado de maneira efetiva um PGRSS com envolvimento de todos os funcionários da equipe multiprofissional. A falta desse gerenciamento pode gerar inúmeros acidentes ou transmitir algum tipo de doença, e, por esse motivo é necessário a existência de programas e políticas públicas a fim de sanar os problemas causados pela produção de resíduos, pois dessa forma a população ganha mais qualidade de vida e fortalece a relação do homem com o meio ambiente (FONSECA et al., 2013).

Sendo assim, o gerenciamento dos RSS associado a capacitação do pessoal, é fundamental para o conhecimento dos procedimentos relacionados a seu manejo e os possíveis riscos à saúde humana e ao meio ambiente (TIVIROLLI et al., 2010). Essa capacitação deve ser direcionada em especial para a educação ambiental, pois segundo Silva et al. (2012) é importante trabalhar esta temática com todo e qualquer cidadão a fim de valorizar sua responsabilidade individual e coletiva, em relação ao meio ambiente.

A educação ambiental deve estar pautada na reflexão, valorizando o ser humano quanto a sua responsabilidade social, bem como sua produção na qualidade de vida, com ações vinculadas a projetos cooperativos concretos, baseados na Política Nacional de Resíduos Sólidos, que incentiva o desenvolvimento de cooperativas e outras formas de associações de catadores como forma e de uma ação socioambiental (FEITOSA et al., 2015).

Após a verificação da quantidade volumétrica dos resíduos, separados por grupos, foi pesquisado o número de leitos da instituição, que também variou no decorrer dos anos, conforme demonstrado na Tabela 2: 
Tabela 2 - Quantidade de leitos hospitalares disponíveis entre os anos de 2010 e 2014

\begin{tabular}{cccccc}
\hline Anos & $\mathbf{2 0 1 0}$ & $\mathbf{2 0 1 1}$ & $\mathbf{2 0 1 2}$ & $\mathbf{2 0 1 3}$ & $\mathbf{2 0 1 4}$ \\
Quantidade de Leitos & 155 & 153 & 198 & 196 & 205 \\
\hline
\end{tabular}

Realizado pelos autores baseado nos PGRSS dos últimos cinco anos da instituição pesquisada

Considerando a Associação Brasileira de Normas Técnicas (ABNT) NBR 10007, que se refere a "amostragem de resíduos sólidos” (ABNT, 2004) e Alcântara (2010), a composição gravimétrica dos resíduos sólidos apresenta porcentagens de várias frações (taxas) dos materiais constituintes dos resíduos em relação ao peso total da amostra estudada.

A partir da aplicação da Taxa Gravimétrica de RSS, construiu-se a Tabela 3 com os resultados dessa taxa entre os anos 2010 e 2014:

Tabela 3 - Taxa gravimétrica dos RSS entre os anos de 2010 e 2014.

\begin{tabular}{lccccc}
\hline Anos & $\mathbf{2 0 1 0}$ & $\mathbf{2 0 1 1}$ & $\mathbf{2 0 1 2}$ & $\mathbf{2 0 1 3}$ & $\mathbf{2 0 1 4}$ \\
Gravimetria RSS/(leitos*dias) & $1,57 \mathrm{Kg}$ & $1,70 \mathrm{Kg}$ & $1,57 \mathrm{Kg}$ & $1,86 \mathrm{Kg}$ & $1,98 \mathrm{Kg}$ \\
\hline
\end{tabular}

Realizado pelos autores baseado nos PGRSS dos últimos cinco anos da instituição pesquisada

É possível perceber que a taxa de geração de resíduos aumentou durante os anos, que variou de $1,57 \mathrm{Kg}$ a $1,98 \mathrm{Kg}$ (leito.ocupado.dia) ${ }^{-1}, \mathrm{e}$, apresentou uma taxa média de $1,73 \mathrm{Kg}$ (leito.ocupado.dia) ${ }^{-1}$.

No Brasil a taxa média de geração de RSS varia de 1,2 a $3,8 \mathrm{Kg}$ (leito.ocupado.dia) $)^{-1}$, enquanto que, na Alemanha, Holanda, Canadá e Áustria, são gerados de 0,005 a $0,4 \mathrm{Kg}$ (leito.ocupado.dia) $^{-1}$ (TIVIROLLI et al., 2010), portanto, as taxas médias de geração de RSS aferidas no presente trabalho, se enquadram nas faixas de valores descritos na literatura.

Um estudo desenvolvido em seis hospitais de VitóriaES, a taxa de geração de resíduos foi uma média de $2,86 \mathrm{Kg}$ (leito.ocupado.dia) $^{-1}$, variando entre 1,37 a $5,34 \mathrm{~kg}$ (leito.ocupado.dia) $)^{-1}$ (ADUAN et al., 2014).

Outro estudo desenvolvido em três hospitais públicos de Mato Grosso do Sul, sendo dois de grande porte e um de pequeno porte, apresentou uma taxa muito maior, devido diversas falhas no gerenciamento dos RSS. As taxas gravimétricas médias foram $4,7 \mathrm{Kg} ; 4,8 \mathrm{Kg} ;$ e $2,4 \mathrm{Kg}$

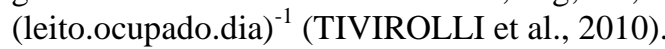

O cálculo da composição gravimétrica dos resíduos geradas por um município, favorece a construção de mais aterros sanitários na região, e, consequentemente maior aproveitamento de resíduos recicláveis (MARTINS et al., 2014)

Segundo a RDC ANVISA $\mathrm{n}^{\circ}$ 306/2004, todos os resíduos do Grupo D encontrados nesta pesquisa poderiam ter sido disposto, em aterro sanitário devidamente licenciado, com redução de custos em relação ao tratamento, contudo alguns deles são misturados a resíduos do Grupo A, o que acaba o sendo considerado infectante como um todo, aumentando assim a proporção de resíduos infectantes, consequentemente, gerando mais gasto para a instituição.

Com base na taxa de geração de RSS sendo 2,68 kg.(leito.ocupado.dia) ${ }^{-1}$, foi estimada uma geração de $1.038,48$ t.ano $^{-1}$ de RSS no ano de 2009, na pesquisa de Aduan et al (2014). A segregação dos resíduos e a melhor escolha dos sistemas de destinação resultariam em uma economia de $93 \%$ nos gastos, quando comparado com o modelo vigente.

Como análise da gestão dos RSS é de fundamental importância o desenvolvimento de práticas cada vez mais sustentáveis que resultam na minimização dos custos com tratamento, bem como a redução do conteúdo para disposição final dos resíduos.
Além da redução de gastos com o tratamento desses resíduos, é possível diminuir os agravos à saúde da população e ao meio ambiente, quando gerados e manejados de maneira adequada. Desta forma evitaria a poluição biológica, física e química do solo, água e do ar, adicionado ao contato direto ou indireto com vetores biológicos e mecânicos (FONSECA et al., 2013).

Como proposta para o planejamento de melhorias no gerenciamento dos RSS, visando a redução de custos, riscos e à preservação do meio ambiente, foram descritas as seguintes sugestões: melhorar a segregação na fonte de RSS que minimize as condições de riscos ambientais e ocupacionais; criar monitoramento periódico de indicadores, como análise gravimétrica mensal dos RSS; reduzir a mistura de resíduos do Grupo D com outros grupos através de medidas de educação continuada para funcionários, enfatizando que parte desse resíduo é reciclável; incentivar a práticas de redução ao desperdício alimentar, diminuindo assim a quantidade de resíduo a ser destinado ao serviço de coleta pública urbana.

Baseado no Manual de Gerenciamento de RSS foi possível fazer uma adaptação para o cálculo da Taxa Gravimétrica de Resíduo de Serviço de Saúde, demonstrando uma variação no decorrer dos cinco anos analisados: $1,57 \mathrm{Kg}$ em $2010 ; 1,70 \mathrm{Kg}$ em $2011 ; 1,57 \mathrm{Kg}$ em $2012 ; 1,86 \mathrm{Kg}$ em 2013 e 1,98Kg.(leito.ocupado.dia) ${ }^{-1}$ em 2014; com uma taxa média de $1,73 \mathrm{Kg}$.(leito.ocupado.dia) ${ }^{-1}$ nos cinco anos analisados. A pesquisa demonstrou que a análise gravimétrica da instituição onde foi realizado o estudo apresentou valores das taxas dentro dos parâmetros esperados, quando comparada a outros dois estudos nacionais.

Como sugestões para melhoria no gerenciamento dos RSS, deve-se ter maior comprometimento com as etapas de manejo dos RSS, realizar análise gravimétrica de RSS periodicamente, reduzir mistura dos diferentes grupos de resíduos, intensificar a educação continuada para os trabalhadores da instituição e aumentar conscientização contra o desperdício alimentar que ocorre na instituição pesquisada.

Com a mudança de atitude de todos os profissionais envolvidos no processo de gerenciamento de RSS será possível uma melhor a qualidade de atendimento à saúde da população, bem como a qualidade ambiental, através da redução de geração e segregação adequada dos resíduos. 


\section{CONCLUSÕES}

De acordo com os dados obtidos neste estudo, pode-se observar as quantidades médias de RSS durante os anos de 2010 a 2014, apresentaram os seguintes resultados: Grupo $\mathrm{A}=29.696,06 \mathrm{Kg} \quad(25,57 \%) ;$ Grupo $\mathrm{B}=28,22 \mathrm{Kg} \quad(0,02 \%)$, Grupo $\mathrm{D}=82.203,94 \mathrm{Kg}(70,79 \%)$ e Grupo $\mathrm{E}=4.200,83 \mathrm{Kg}$ $(3,62 \%)$.

A partir dos pesos médios de cada grupo de resíduos, o do Grupo D destacou-se por sua elevada quantidade, podendo ser resultado de desperdício alimentar e resíduos recicláveis, porém não aproveitados. Outro fato de relevante importância do risco de misturar os resíduos do Grupo A com outros grupos de resíduos, tornando todo o montante potencialmente infectante, gerando maiores custo com o tratamento e destinação final.

\section{REFERÊNCIAS BIBLIOGRÁFICAS}

ADUAN, S.A.; BRAGA, F.S.; ZANDONADE, E.; SALLES, D.; CUSSIOL, N.A.M.; LANGE, L.C. Avaliação dos RSS do Grupo A em Hospitais de Vitória (ES), Brasil. Rev. Eng. Sanit. Ambient, v.19, n.2, p.133-144, 2014.

AGÊNCIA NACIONAL DE VIGILÂNCIA SANITÁRIA. ANVISA. Resolução RDC no 306 de 7 de dezembro de 2004. Dispõe sobre o regulamento técnico para o gerenciamento de resíduos de serviços de saúde. Diário Oficial da União, 2004.

ALCÂNTARA, A.J.O. Composição gravimétrica dos resíduos sólidos urbanos e caracterização química do solo da área de disposição final do município de Cáceres-MT. Cáceres, 2010. Dissertação (Mestrado em Ciências Ambientais) - Universidade do Estado de Mato Grosso, Cárceres, 2010.

ASSOCIAÇÃO BRASILEIRA DE EMPRESAS DE LIMPEZA PÚBLICA E RESÍDUOS ESPECIAIS ABRELPE. Panorama dos Resíduos Sólidos no Brasil, 2014.

ASSOCIAÇÃO BRASILEIRA DE NORMAS TÉCNICAS. ABNT. NBR 10007: 2004. Amostragem de resíduos sólidos. Rio de Janeiro: ABNT, 2004.

BRASIL. Ministério da Saúde. Biblioteca Virtual em Saúde. Secretaria Executiva. Manual de gerenciamento de resíduos de serviços de saúde. Brasília: Ministério da Saúde, 2001. Disponível em: <http://bvsms.saude.gov.br/bvs/publicacoes/manual_ger enciamento_residuos.pdf>. Acesso em: 22 out. 2015.

CONSELHO NACIONAL DE MEIO AMBIENTE. CONAMA. Resolução no 358 de 29 de abril de 2005. Dispõe sobre o tratamento e a disposição final dos resíduos dos serviços de saúde e dá outras providências. Diário Oficial da União, 2005.

COSTA, E.C.L. Manejo de resíduos de serviços de saúde: manual básico de procedimentos. Brasília: Câmara dos Deputados, Edições Câmara, 2012.

FEITOSA, A.K.; LANDIM, G.H.P.; SILVA, K. B.; SILVA, G.D. A Educação Cooperativista na Formação de Catadores de Resíduos Sólidos em Iguatu, Ceará.
Revista Verde de Agroecologia e Desenvolvimento Sustentável (Pombal - PB - Brasil), v. 10, n.3, p.09-13, 2015.

FONSECA, M.D.; CARVALHO, G.G.; CORRÊA, M.M.; HOLANDA, R.M. Os riscos relacionados ao meio ambiente e à atividade de coleta de resíduos sólidos urbanos. Revista Verde de Agroecologia e Desenvolvimento Sustentável (Mossoró - RN BRASIL), v.8, n.5, p.96-100, 2013.

FUNDAÇÃO ESTADUAL DO MEIO AMBIENTE. FEAM. Manual de gerenciamento de resíduos de serviços de Saúde. Belo Horizonte: FEAM, 2008.

INSTITUTO BRASILEIRO DE GEOGRAFIA E ESTATÍSTICA. IBGE. Censo demográfico. Rio de janeiro: IBGE, 2012.

MARTINS, W.A.; ALBUQUERQUE, W.G.; NUNES, F.M.S.; ALMEIDA, R.N.; COSTA, F.F. Gerenciamento dos resíduos sólidos urbanos no município de pombal PB - Brasil. Revista Verde de Agroecologia e Desenvolvimento Sustentável (Mossoró - RN BRASIL), v. 9, n.1, p. 89-97, 2014.

MOURA, A.A.; LIMA, W.S.; ARCHANJO, C.R. Análise da composição gravimétrica de resíduos sólidos urbanos: estudo de caso - município de Itaúna-MG. SynThesis Revista Digital FAPAM, Pará de Minas, n.3, p.4-16, 2012.

SES/MT. SECRETARIA DE ESTADO DE SAÚDE DE MATO GROSSO. Governo de Mato Grosso. Disponível em: < http://www.saude.mt.gov.br/> Acesso em: 10 out. 2015.

SILVA, G.F.; SILVA, M.R.F.; DIAS, N.S.; NETO, M.F.; PIMENTA, M.R.C. Educação Ambiental: Diagnóstico de práticas ambientais no projeto de assentamento Milagres, Apodi/RN. Revista Verde de Agroecologia e Desenvolvimento Sustentável (Mossoró - RN), v.7, n.7, p.01-07, 2012.

SOUSA JÚNIOR, F. S.; SOUZA, L.D.; CAMACHO, R.G.V.; EVAGELISTA, J.P.C.; SILVA, N.F. Educação ambiental e gestão dos resíduos sólidos da Associação Reciclando para a Vida - ACREVI, Mossoró - RN. Revista Verde de Agroecologia e Desenvolvimento Sustentável (Mossoró - RN - Brasil), v.3, n.2, p. 64-71, 2008.

SOUSA, K.S.; DANTAS, I.A.M.; SÁ, G.B.; ANDRADE, A.B.A.; MARACAJÁ, P.B. Gerenciamento dos resíduos sólidos urbanos no município de São Bentinho-PB Brasil. Revista Verde de Agroecologia e Desenvolvimento Sustentável (Pombal - PB - Brasil), v.10, n.3, p.13-19, 2015.

TIVIROLLI, K.; TIVIROLLI, S.C.; LUZ, P.C.; FUJINO, L.B.V.; SHINZATO, M.P.; SKOWRONSKI, J.; GOMES, A.O.; VASCONCELOS, L.H.A.; HESS, S.C. Gerenciamento dos resíduos em três hospitais públicos no Mato Grosso do Sul, Brasil. RBPS, v.23, n.3, p.213220. 